\title{
Unusual presentation of Herpes Simplex Encephalitis:A Case Study.
}

\author{
${ }^{1}$ Dr.Vijaykumar S. Gulwe, ${ }^{2}$ Dr.Vishal R. Dalvi, ${ }^{3}$ Dr.Vitthal Pawar, ${ }^{4}$ Dr.Bhushan \\ Pagar, ${ }^{5}$ Dr.Rushikesh Patil, ${ }^{6}$ Dr.Preetam Ahire. \\ ${ }^{1}$ Senior Professor, ${ }^{2}$ Chief Resident In Medicine, ${ }^{3}$ Chief Resident In Medicine, ${ }^{4}$ Senior Resident In Medicine, \\ ${ }^{5}$ Senior Resident In Medicine, ${ }^{6}$ Junior Resident in Medicine \\ Department of Medicine, Mahatma Gandhi Mission's Medical College \& Hospital, CIDCO, N-6, Aurangabad, \\ Maharashtra, India - 431003.
}

\begin{abstract}
We report a case of Herpes Simplex Encephalitis presenting with fever, seizure, headache, vomiting, memory loss, confusion which is indeed a very unsual and rare presentation. She recovered very well with antiviral and anti epileptic medications. Herpes simplex encephalitis (HSE) is an acute or subacute illness that causes both general and focal signs of cerebral dysfunction. Brain infection is thought to occur by means of direct neuronal transmission of the virus from a peripheral site to the brain via the trigeminal or olfactory nerve. The exact pathogenesis is unclear, and factors that precipitate HSE are unknown. Diagnosing this disease early \& providing appropriate treatment with antiviral therapy is important to achieve an optimal clinical outcome. Early administration of antiviral therapy is the only parameter that can be modified to improve the prognosis of patients with HSE.
\end{abstract}

Key words: HSE, memory loss, antiviral therapy

\section{Introduction}

Herpes simplex encephalitis (HSE) is the most common sporadic necrotizing encephalitis in the Western world ${ }^{1}$. HSE is a life-threatening consequence of herpes simplex virus (HSV) infection of the central nervous system (CNS). HSE is a rare, but severe viral infection of the human central nervous system. It is estimated to affect at least 1 in 500,000 individuals per year ${ }^{2}$ and some studies suggest an incidence rate of 5.9 cases per 100,000 live births. ${ }^{3}$ The majority of cases of herpes encephalitis are caused by herpes simplex virus-1 (HSV-1), the same virus that causes cold sores. 57\% of American adults are infected with HSV-1, ${ }^{4}$ which is spread through droplets, casual contact and sometimes sexual contact, though most infected people never have cold sores. About $10 \%$ of cases of herpes encephalitis are due to HSV-2, which is typically spread through sexual contact. About 1 in 3 cases of HSE result from primary HSV-1 infection, predominantly occurring in individuals under the age of $18 ; 2$ in 3 cases occur in seropositive persons, few of whom have history of recurrent orofacial herpes. Approximately $50 \%$ of individuals that develop HSE are over 50 years of age. ${ }^{5}$

\section{Case Report}

A 40 years old female patient presented to emergency room with complaints of fever, headache, vomiting, ,one episode of generalized tonic clonic seizure, memory loss (recent and past) and confusion.

On clinical examination, the patient was obese and well nourished. Patient was lethargic, disoriented, febrile with a pulse of 106/min, regular in rhythm. Blood pressure was $130 / 80 \mathrm{~mm}$ of $\mathrm{Hg}$. Cardiovascular examination revealed tachycardia. Respiratory system \& per abdomen examination was within normal limits.CNS examination revealed Glassgow Coma Scale (GCS) 14/15, no neck rigidity, all reflexes intact, normal tone and power, left plantar extensor and right flexor, pupils normal sized \& bilaterally reacting to light. Fundus examination revealed papilloedema. Hence CSF study was not done. However, papilloedema resolved gradually by intravenous mannitol $(0.5 \mathrm{~g} / \mathrm{kg} / \mathrm{day} 8 \mathrm{hrly}$ for 5 days $)$. CT Scan Brain revealed no significant abnormality. MRI Brain study revealed swelling of right medial temporal lobe, parahippocampal gyrus, insular cortex with restricted diffusion in bilateral medial temporal lobes,insular cortices and cingulated gyri. MR Venography and EEG revealed no significant abnormality. Details of investigations done during hospitalization are described in the table below(TABLE 1). Patient was treated as a case of Herpes Simplex Encephalitis with intravenous antiviral therapy of acyclovir (30 $\mathrm{mg} / \mathrm{kg} / \mathrm{day}$ 6hrly for 10 days), antiepileptic medication in the form of intravenous phenytoin $(3 \mathrm{mg} / \mathrm{kg} /$ day 8 hrly for 3days).

Patient made a good recovery after antiviral therapy with improvement in her memory status (past and recent) and was subsequently discharged home 15 days, post admission.MRI Brain was repeated at the time of discharge and was compared with the previous one which revealed mild regression of left temporal lobe signal with mild progression of altered signal in right temporal lobe. Patient was discharged on oral antiviral drug 
(Tablet Acyclovir 800mg QID for 10 days),antiepileptic (Tablet levetirecetam 500mg BD till medical advise) and to be followed up after 15 days.

\section{Discussion}

Herpes simplex encephalitis (HSE) is a rare neurological disorder characterized by inflammation of the brain (encephalitis). Patients with HSE may have a prodrome of malaise, fever, headache, and nausea, followed by acute or subacute onset of an encephalopathy whose symptoms include lethargy, confusion, and delirium. However, no pathognomonic clinical findings reliably distinguish HSE from other neurologic disorders with similar presentations. ${ }^{6}$

The following are typically the most common symptoms of $\mathrm{HSE}^{7}$ :

- $\quad$ Fever $(90 \%)$

- Headache $(81 \%)$

- Psychiatric symptoms $(71 \%)$

- Seizures $(67 \%)$

- Vomiting $(46 \%)$

- Focal weakness (33\%)

- Memory loss (24\%)

The initial presentation may be mild or atypical in immunocompromised patients (eg, those with HIV infection or those receiving steroid therapy). ${ }^{8}$

HSE is thought to be caused by the retrograde transmission of virus from a peripheral site on the face following HSV-1 reactivation, along a nerve axon, to the brain. ${ }^{9}$ The virus lies dormant in the ganglion of the trigeminal cranial nerve, but the reason for reactivation, and its pathway to gain access to the brain, remains unclear, though changes in the immune system caused by stress clearly play a role in animal models of the disease. The olfactory nerve may also be involved in HSE, ${ }^{10}$ which may explain its predilection for the temporal lobes of the brain, as the olfactory nerve sends branches there. In horses, a single-nucleotide polymorphism is sufficient to allow the virus to cause neurological disease, ${ }^{11}$ but no similar mechanism has been found in humans.

The most frequent findings on physical examination are fever and mental status abnormalities. Meningeal signs may be present, but meningismus is uncommon.

Typical findings on presentation include the following ${ }^{12}$ :

- Alteration of consciousness (97\%)

- Fever $(92 \%)$

- Dysphasia $(76 \%)$

- Ataxia (40\%)

- Seizures (38\%) - Focal (28\%); generalized (10\%)

- Hemiparesis (38\%)

- Cranial nerve defects $(32 \%)$

- Visual field loss (14\%)

- Papilloedema (14\%)

A causal or temporal relationship between peripheral lesions (eg, herpes labialis) and HSE does not exist. In addition, many febrile diseases may precipitate herpes labialis. Therefore, the presence or absence of such lesions neither confirms nor excludes the diagnosis.

Unusual presentations occur. Both herpes simplex virus type 1 (HSV-1) and herpes simplex virus type 2 (HSV-2) may produce a more subacute encephalitis, apparent psychiatric syndromes, and benign recurrent meningitis. Less commonly, HSV-1 may produce a brain stem encephalitis, and HSV-2 may produce a myelitis.

Anterograde memory often is impaired even with successful treatment of HSE. Retrograde memory, executive function, and language ability also may be impaired. A study by Utley et al showed that patients who had a shorter delay $\left(<5\right.$ days) between presentation and treatment had better cognitive outcomes. ${ }^{13}$

$\mathrm{Ku}$ et al discussed the unique presentation of HSE in a bilingual patient, who developed global aphasia for 1 language (his most recently learned language) but retained most of his birth language ability. ${ }^{14}$

McGrath et al reported on 4 patients with confirmed HSE, each with an anterior opercular syndrome, and observed that the syndrome (ie, paralysis of the masticatory, facial, pharyngeal, and lingual muscles) occurred as the primary manifestation of HSE in 2 patients and as part of the encephalitis picture in the other 2 patients. ${ }^{15}$ The authors suggested that unique presentations (eg, anterior opercular syndrome), should alert the clinician to the possibility of HSE.

Mondal et al reported basal ganglia involvement in a child with HSE, demonstrating extrapyramidal symptoms. ${ }^{16} \mathrm{Li}$ and Sax reported HSE-associated cerebral hemorrhage in an HIV-positive person. ${ }^{17}$

Subha Priya Venkateshwaran, Kamaraj Murugesan and Rajeshwari Sivaraj 2011 studied Seroprevalence of Ig G and Ig M Antibodies in Individuals with Herpes Simplex Virus -1 \&2 Infection in HIV 
Positive and Negative Individuals of South Indian Population .31 out of the 35 HIV positive samples and 32 out of the 35 HIV negative samples tested positive for HSV antibodies. In that $34.3 \%$ of both HIV positive and negative individuals were positive for HSV-1 IgM antibodies. $80 \%$ of HIV positive individuals and $71.4 \%$ of HIV negative individuals were positive for HSV-1 IgG antibodies. $28.6 \%$ of HIV positive individuals and $17.1 \%$ of HIV negative individuals were positive for HSV-2 IgM antibodies. $60 \%$ of HIV positive and $17.1 \%$ of HIV negative individuals was positive for HSV-2 IgG antibodies. ${ }^{18}$ (Table 2).

Untreated HSE is progressive and often fatal in 7-14 days. A landmark study by Whitley et al in 1977 revealed $70 \%$ mortality in untreated patients and severe neurologic deficits in most of the survivors. ${ }^{19}$

MRI scan - The activity of the brain changes as the disease progresses, first showing abnormalities in one temporal lobe of the brain, which spread to the other temporal lobe 7-10 days later. ${ }^{20}$ : MRI is more sensitive than CT scan and is now the imaging study of choice. ${ }^{21}$

Electroencephalogram (EEG): shows abnormalities in about $80 \%$ of cases of HSE. These include focal temporal changes, diffuse slowing, periodic complexes and periodic lateralising epileptiform discharges.

Definite diagnosis requires testing of the cerebrospinal fluid (CSF) by a lumbar puncture (spinal tap) for presence of the virus.

Lumbar puncture:Only after a mass-effect lesion has been excluded by imaging.Mildly elevated protein, normal or slightly low glucose, and a moderate pleocytosis (mostly mononuclear cells). Red blood cells and xanthochromia may be seen.

Herpes infection can usually be confirmed by identification of virus in CSF by a polymerase chain reaction (PCR), which is the diagnostic test of choice to detect HSV DNA. PCR is highly specific but false negatives can occur early after disease onset. ${ }^{22}$

Brain biopsy: Allows identification of HSV in brain tissue but is rarely indicated because of effective methods of diagnosis and relatively non-toxic and effective antiviral medications.

The testing takes several days to perform, and patients with suspected Herpes encephalitis should be treated with acyclovir immediately while waiting for test results.

The age of the patient and the level of consciousness at initiation of therapy have been identified as major determinants of prognosis ${ }^{23}$. More recently, the delay between admission of the patient to the hospital and initiation of acyclovir was identified as a main predictor of outcome ${ }^{24}$

TABLE 1

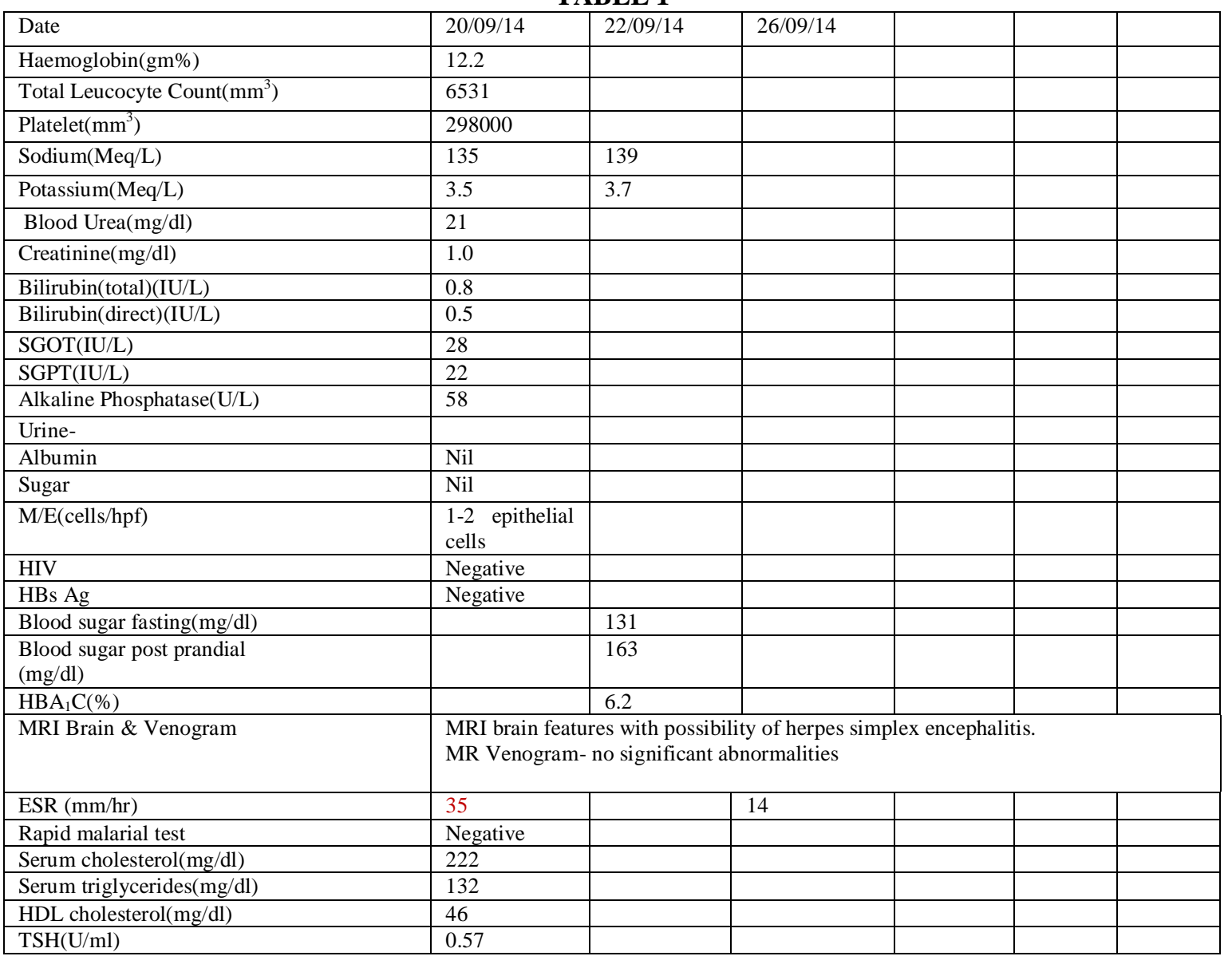


Unusual presentation of Herpes Simplex Encephalitis:A Case Study.

Table 2

\begin{tabular}{|l|l|l|l|l|l|}
\hline & & \multicolumn{1}{|c|}{ HIV Positive samples } & \multicolumn{2}{c|}{ HIV Negative samples } \\
\hline Assay Types & Antibodies & $\begin{array}{l}\text { Number Of } \\
\text { Positives }\end{array}$ & Percentage & $\begin{array}{l}\text { Number } \\
\text { Positives }\end{array}$ & Percentage \\
\hline HSV 1 & 12 & 28 & 34.3 & 12 & 34.3 \\
\hline ELISA & IgM & 10 & 80 & 25 & 71.4 \\
\hline HSV 2 & IgG & 21 & 60 & 6 & 17.1 \\
\hline ELISA & IgM & IgG & & 6 & 6 \\
\hline
\end{tabular}
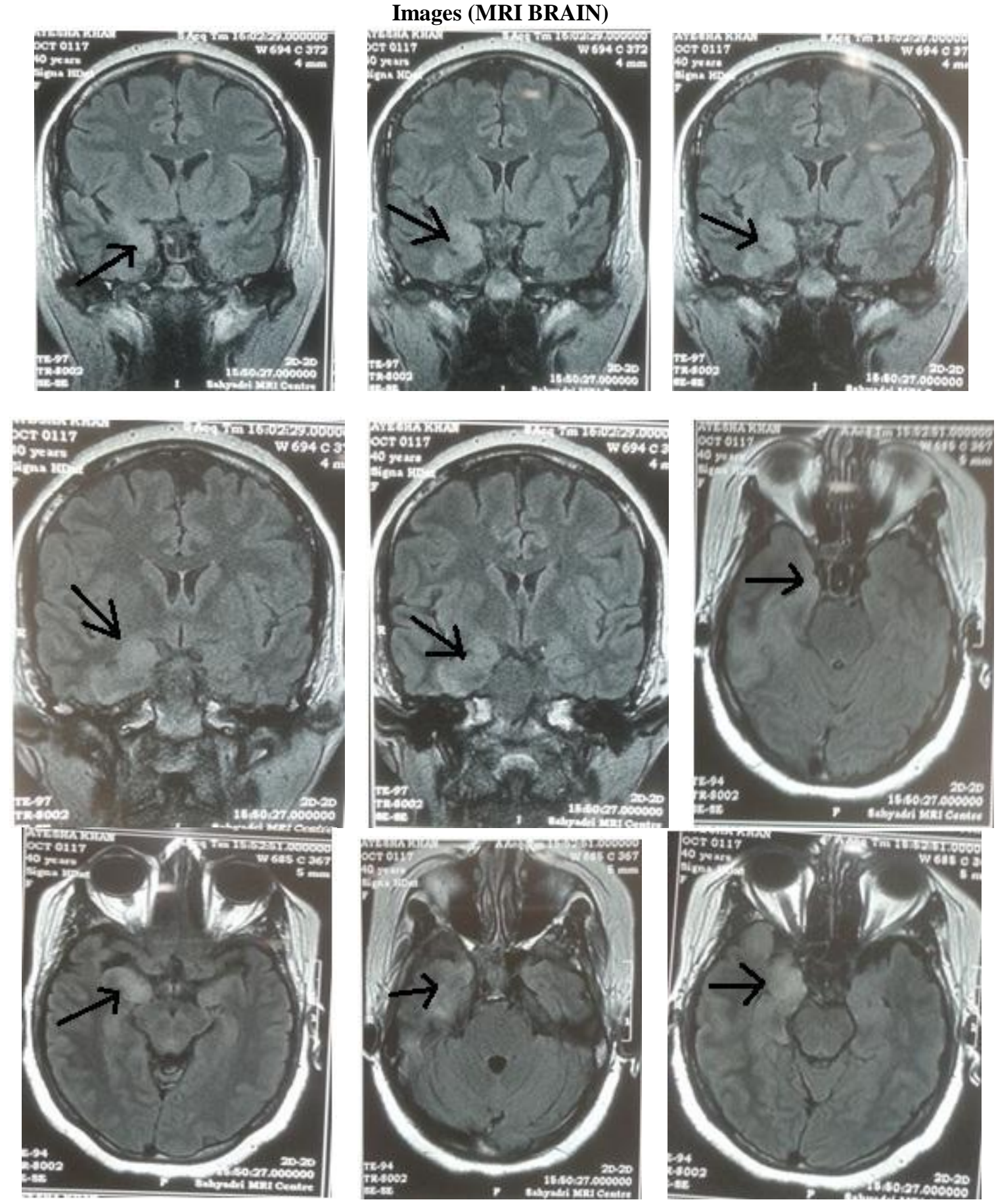

MRI Brain study showing swelling of right medial temporal lobe, parahippocampal gyrus, insular cortex with restricted diffusion in bilateral medial temporal lobes,insular cortices and cingulated gyri. 


\section{Conclusion}

Patients presenting with fever, headache, vomiting, seizure, memory loss (recent and past) and confusion should be suspected for Herpes encephalitis \& should be treated with acyclovir immediately to avoid complications like behavioral/personality changes, language and speech problems, intellectual disability,vision problems, respiratory distress, as testing takes several days to perform . Therefore, taking into consideration, the high mortality and morbidity associated with focal encephalitis, poor feasibility of definitive diagnostic tests, and our experience with intravenous acyclovir, we feel that this modality of treatment has potentials to prove beneficial,if initiated during early phase of HSE. However taking into consideration its cost effectiveness, there is still a pressing need to develop yet more effective treatments for HSE.

\section{References}

[1]. Whitley RJ. Viral encephalitis. N Engl J Med 1990;323:242-50.

[2]. Whitley RJ (2006). "Herpes simplex encephalitis: adolescents and adults". Antiviral Res. 71 (2-3): 14

[3]. Kropp, Rhonda Y., et al. (2006). "Neonatal herpes simplex virus infections in Canada: results of a 3 -year national prospective study.". Pediatrics Vis. Sci. 117 (6): 1955-1962.

[4]. Xu, Fujie; Sternburg Maya; Kottiri Benny; McQuilan Geraldine; Lee Francis; Nahmias Andre; Berman Stuart; Markowitz Larui (2006). "Trends in Herpes Simplex Virus Type 1 and Type 2 Seroprevalence in the United States". JAMA. 8 296 (8): $964-73$

[5]. RJ, Gnann JW (2002). "Viral encephalitis: familiar infections and emerging pathogens". Lancet 359 (9305): 507-13.

[6]. Whitley RJ, Cobbs CG, Alford CA Jr, Soong SJ, Hirsch MS, Connor JD, et al. Diseases that mimic herpes simplex encephalitis. Diagnosis, presentation, and outcome. NIAD Collaborative Antiviral Study Group.JAMA. Jul 14 1989;262(2):234-9.

[7]. Whitley RJ, Soong SJ, Linneman C Jr, Liu C, Pazin G, Alford CA. Herpes simplex encephalitis. Clinical Assessment. JAMA. Jan 15 1982;247(3):317-20.

[8]. Tan IL, McArthur JC, Venkatesan A, Nath A. Atypical manifestations and poor outcome of herpes simplex encephalitis in the immunocompromised. Neurology. Nov 20 2012;79(21):2125-32.

[9]. Whitley RJ (2006). "Herpes simplex encephalitis: adolescents and adults". Antiviral Res. 71 (2-3): 141-8.

[10]. Dinn J (1980). "Transolfactory spread of virus in herpes simplex encephalitis". Br Med J 281 (6252): 1392.

[11]. Van de Walle GR, Goupil R, Wishon C, et al. (2009). "A single-nucleotide polymorphism in a herpesvirus DNA polymerase is sufficient to cause lethal neurological disease". J Infect Dis 200 (1): 20-25.

[12]. Whitley RJ, Soong SJ, Linneman C Jr, Liu C, Pazin G, Alford CA. Herpes simplex encephalitis. Clinical Assessment. JAMA. Jan 15 1982;247(3):317-20

[13]. Utley TF, Ogden JA, Gibb A, McGrath N, Anderson NE. The long-term neuropsychological outcome of herpes simplex encephalitis in a series of unselected survivors. Neuropsychiatry Neuropsychol Behav Neurol. Jul 1997;10(3):180-9.

[14]. Ku A, Lachmann EA, Nagler W. Selective language aphasia from herpes simplex encephalitis. Pediatr Neurol. Sep 1996;15(2):16971.

[15]. McGrath NM, Anderson NE, Hope JK, Croxson MC, Powell KF. Anterior opercular syndrome, caused by herpes simplex encephalitis. Neurology. Aug 1997;49(2):494-7.

[16]. Mondal G, Kumar R, Ghosh JK, Basu K, Chatterjee S. Basal ganglia involvement in a child with herpes simplex encephalitis. Indian J Pediatr. Jul 2009;76(7):749-50

[17]. Li JZ, Sax PE. HSV-1 encephalitis complicated by cerebral hemorrhage in an HIV-positive person. AIDS Read. Apr 2009;19(4):153-5

[18]. Subha Priya Venkateshwaran, Kamaraj Murugesan and Rajeshwari Sivaraj Seroprevalence of Ig G and Ig M Antibodies in Individuals with Herpes Simplex Virus -1 \&2 Infection in HIV Positive and Negative Individuals of South Indian Population Journal of Applied Pharmaceutical Science 01 (10); 2011: 154-158

[19]. Whitley RJ, Soong SJ, Dolin R, Galasso GJ, Ch'ien LT, Alford CA. Adenine arabinoside therapy of biopsy-proved herpes simplex encephalitis. National Institute of Allergy and Infectious Diseases collaborative antiviral study. N Engl J Med. Aug 11 $1977 ; 297(6): 289-94$.

[20]. Whitley RJ (2006). "Herpes simplex encephalitis: adolescents and adults". Antiviral Res. 71 (2-3): 141-8.

[21]. Sabah M, Mulcahy J, Zeman A; Herpes simplex encephalitis. BMJ. 2012 Jun 6;344

[22]. Whitley RJ, Kimberlin DW; Herpes simplex encephalitis: children and adolescents. Semin Pediatr Infect Dis. 2005 Jan;16(1):17-23.

[23]. Whitley RJ, Alford CA, Hirsch MS, et al. Vidarabine versus acyclovir therapy in herpes simplex encephalitis. N Engl J Med 1986;314:144-9.

[24]. McGrath N, Anderson NE, Croxson MC, Powell KF. Herpes simplex encephalitis treated with acyclovir: diagnosis and long term outcome. J Neurol Neurosurg Psychiatry 1997;63:321-6. 